\title{
Sugar, Pressure and Pregnancy
}

Controlling blood glucose is one of the banes in the life of the intensive care unit (ICU) nurse. The story of tight glucose control started in 2001, when Van den Berghe et al. ${ }^{[1]}$ published the startling finding that maintaining tight glucose control in the range of $4.5-6.0 \mathrm{mmol} / \mathrm{L}$ in a surgical ICU dramatically decreased mortality. ICUs across the world began implementing tight glucose control strategies accordingly, but the expected improved outcome was not realised. This observation was confirmed by the multi-centered NICE-SUGAR trial, ${ }^{[2]}$ which showed that the intervention actually increased mortality and the increased deaths were linked to an unacceptably high incidence of hypoglycaemic events. In this issue of SAJCC, we publish a study by Maharaj et al. ${ }^{[3]}$ showing that protocol violations in blood glucose control in a cardiothoracic ICU are very common and results in wide swings in glucose levels. It appears that unless there is a high ratio of expert nurses available, the tight protocol does more harm than good. ICUs that are still trying to follow the tight range should adjust their targets immediately. The current Surviving Sepsis guidelines recommend that blood glucose be controlled using a protocolised approach: a target of $<10.0 \mathrm{mmol} / \mathrm{L}$ should be maintained and that arterial blood should be used for the measurement if an arterial catheter is in place. ${ }^{[4]}$ This is more easily achievable than tight glucose control and allows nurses more time to perform other, important procedures. Once technology allows us to close the loop between continuous glucose monitoring and insulin pump control, we should consider revisiting tight control.

Arterial catheters are widely used in ICUs for monitoring blood pressure and for arterial blood sampling. The problem of under- and overdamping of the intra-arterial blood pressure monitoring system was first comprehensively studied in 1981, and intensivists and anaesthesiologists were well aware of the problem. ${ }^{[5]}$ Because this phenomenon leads to inaccurate systolic and diastolic readings, while minimally affecting mean blood pressures, we have more recently tended to ignore damping issues and to use mean pressures as the main therapeutic target in managing the critically ill patient. However, the increasing use of the invasive arterial pressure trace for monitoring stroke volume and intravascular volume responsiveness means that we must, once again, pay attention to this issue, as these techniques rely on accurate systolic and diastolic readings, as well as a true depiction of the pressure curve. In this issue of SAJCC, we publish a survey of intra-arterial pressure monitoring systems in an ICU. ${ }^{[6]}$ Only $19 \%$ of systems were appropriately damped, with the rest being over- or under-damped. Overdamping can be corrected by paying attention to details, such as under-pressurised flush bags, blood clots, bubbles, and malpositioned catheters. Underdamping is caused by resonance in the system, which can be controlled by a propriety device inserted between the arterial catheter and the transducer. ${ }^{[7]}$ One solution may be for monitor manufacturers to develop software that could detect the presence of an over- or underdamped system and possibly even correct for this error.

Obstetric patients form a greater proportion of the ICU population in developing countries when compared with developed countries. In this issue, we publish a study by Motiang ${ }^{[8]}$ of 210 obstetric patients admitted to a tertiary-level ICU over a 4 -year period. The patients were young, with an average admission time of 24 hours and a mortality rate of only $9 \%$. The most common reason for ICU admission was pre-existing cardiac disease and the second most frequent reason was preeclampsia, which was probably the reason for the main cause of death - intracerebral haemorrhage. This study suggests that obstetric patients are worthwhile occupiers of ICU beds and systems should be in place for them to have rapid access to critical care when it is required.

The problem of abdominal hypertension and the development of abdominal compartment syndrome is now well recognised, but the accurate measurement of intra-abdominal pressure is crucial for the diagnosis. Bladder catheters are usually used, as direct measurement is too invasive. It is important to strictly follow international guidelines to ensure reliable readings. ${ }^{[9]}$ For a variety of reasons, where the transvesical route cannot be used, the transgastric route is recommended. The animal model published in this issue is reassuring, as it confirms that there are no significant differences between the three methods of intra-abdominal pressure measurement in two different abdominal hypertension models. ${ }^{[10]}$

Finally, I recommend reading Nankundwa's ${ }^{[11]}$ qualitative study on the emotional response of nurses caring for patients with do-not-resuscitate orders. Poor communication between the medical and nursing staff was reported to be the main source of emotional distress. As one subject stated, 'Usually the decision is made by doctors and nurses are the ones to implement it'. This highlights the importance of honest, open communication and respect among healthcare professionals, including doctors and nurses, working in critical care - for the benefit of both patients and staff.

\section{W Lance Michell}

Editor

lance.michell@uct.ac.za

\section{S Afr J Crit Care 2017;33(1):2. DOI:10.7196/SAJCC.2017.v33i1.337}

1. Van den Berghe G, Wouters P, Weekers F, et al. Intensive insulin therapy in critically ill patients. N Engl J Med 2001;345(19):1359-1367. https://doi.org/10.1056/nejmoa011300

2. The NICE-SUGAR Study Investigators. Intensive versus conventional glucose control in critically ill patients. N Engl J Med 2009;360(13):1283-1297. https://doi.org/10.1056/nejmoa0810625

3. Maharaj D, Perrie H, Scribante J, Paruk F. Glycaemic control in a cardiothoracic surgical population: Exploring the protocol-practice gap. S Afr J Crit Care 2017;33(1):4-7. https://doi org/10.7196/SAJCC.2017.v33i1.280

4. Rhodes A, Evan LE, Alhazzani W, et al. Surviving Sepsis Campaign: International guidelines for management of sepsis and septic shock: 2016. Intensive Care Med 2017;43(3):304-377. https://doi. org/10.1007/s00134-017-4683-6

5. Gardner RM. Direct blood pressure measurement - dynamic response requirements. Anesthesiol 1981;54(3):227-236. https://doi.org/10.1097/00000542-198103000-00010

6. Rooke WH, Turner JD, Clutton-Brock TH. Analysis of damping characteristics of arterial catheter blood pressure monitoring in a large intensive care unit. S Afr J Crit Care 2017;33(1):8-10. https:// doi.org/10.7196/SAJCC.2017.v33i1.300

7. Todorovic M, Jensen EW, Thøgersen C. Evaluation of dynamic performance in liquid-filled catheter systems for measuring invasive blood pressure. Int J Clin Monit Comput 1996;13(3):173178. https://doi.org/10.1023/a:1016903508976

8. Motiang M. Obstetric patients admitted to intensive care unit of Dr George Mukhari Academic Hospital, Ga-Rankuwa, South Africa. S Afr J Crit Care 2017;33(1):12-14. https://doi.org/10.7196/ SAJCC.2017.v33i1.281

9. Kirkpatrick AW, Roberts DJ, De Waele J, et al. Intra-abdominal hypertension and the abdominal compartment syndrome: Updated consensus definitions and clinical practice guidelines from the World Society of the Abdominal Compartment Syndrome. Intensive Care Med 2013,39(7):11901206. https://doi.org/10.1007/s00134-013-2906-z

10. Wise RD, Rodseth RN, Correa-Martin L, et al. Correlation between different methods of intraabdominal pressure monitoring in varying intra-abdominal hypertension models. S Afr J Crit Care 2017;33(1):15-18. https://doi.org/10.7196/SAJCC.2017.v33i1.327

11. Nankundwa E, Brysiewicz P. Lived experiences of Rwandan ICU nurses caring for patients with a do-not-resuscitate order. S Afr J Crit Care 2017;33(1):19-22. https://doi.org/10.7196/SAJCC.2017. v33i1.281 